\title{
A converse theorem for Dirichlet $L$-functions
}

\author{
Jerzy Kaczorowski, Giuseppe Molteni and Alberto Perelli
}

\begin{abstract}
It is known that the space of solutions (in a suitable class of Dirichlet series with continuation over $\mathbb{C}$ ) of the functional equation of a Dirichlet $L$-function $L(s, \chi)$ has dimension $\geq 2$ as soon as the conductor $q$ of $\chi$ is at least 4 . Hence the Dirichlet $L$-functions are not characterized by their functional equation for $q \geq 4$. Here we characterize the conductors $q$ such that for every primitive character $\chi(\bmod q), L(s, \chi)$ is the only solution with an Euler product in the above space. It turns out that such conductors are of the form $q=2^{a} 3^{b} m$ with any square-free $m$ coprime to 6 and finitely many $a$ and $b$.
\end{abstract}

Mathematics Subject Classification (2000). 11M06, $11 \mathrm{M} 41$.

Keywords. Dirichlet $L$-functions, converse theorems, Hamburger theorem, Selberg class.

\section{Introduction}

A well known theorem by Hamburger, see [7] and Chapter II of Titchmarsh [16], states that the Riemann zeta function $\zeta(s)$ is determined by its functional equation in the following sense. Let $f(s)$ and $g(s)$ be two Dirichlet series absolutely convergent for $\sigma>1$ such that $(s-1) f(s)$ and $(s-1) g(s)$ are entire functions of finite order, and $f(s)$ and $g(s)$ satisfy the functional equation

$$
\pi^{-s / 2} \Gamma\left(\frac{s}{2}\right) f(s)=\pi^{-(1-s) / 2} \Gamma\left(\frac{1-s}{2}\right) g(1-s) .
$$

Then $f(s)=g(s)=c \zeta(s)$ for some $c \in \mathbb{C}$. In fact, the same conclusion holds under weaker conditions on $f(s)$ and $g(s)$; we refer to Piatetski-Shapiro and Raghunathan [15], and to the literature quoted there, for an interesting discussion of the above theorem, especially in connection with uniqueness properties of the Poisson summation formula.

Hamburger's theorem is the first and simplest example of a converse theorem, roughly speaking a result characterizing $L$-functions by means of their standard analytic properties. Several converse theorems are known in the literature. We mention here only the classical converse theorems by Hecke (see Chapter I of [8]) and Weil 
[17] concerning the $L$-functions associated with modular forms (see also ConreyFarmer [4] for an interesting variant involving Euler products), and the general converse theorems for automorphic $L$-functions, see Cogdell and Piatetski-Shapiro [3]. Moreover, a general converse theorem for degree $1 \mathrm{~L}$-functions has been established by Kaczorowski-Perelli [9] in the framework of the Selberg class.

Hamburger's theorem has a special feature among converse theorems. In fact, it shows that the vector space of the Dirichlet series satisfying the functional equation of $\zeta(s)$ and some standard analytic properties is 1-dimensional. This indeed happens rarely, as shown by Theorem 2 of [9] for degree $1 L$-functions: the 1-dimensional case arises only when the conductor equals 1 (essentially Hamburger's theorem) or 3 . In particular, the strict analog of Hamburger's theorem holds, among the Dirichlet $L$-functions with non-trivial character, only when $L(s, \chi)$ is associated with the odd character $\chi(\bmod 3)$. This follows also from the arguments in [15], see p. 117, and essentially also from the third part of Hamburger's original paper. Similar phenomena are expected to hold for higher degree $L$-functions as well.

In view of the above discussion it is natural to address the following question: under what conditions a functional equation has only one solution in the set of Dirichlet $L$-functions? As we shall see in Theorem 1 below, the question essentially asks for an analog of Hamburger's theorem where the Euler product is added to the standard analytic properties. In this paper we characterize the moduli $q$ such that all the functional equations $(\bmod q)$ have only one solution in the set of Dirichlet $L$-functions.

We recall that for a primitive Dirichlet character $\chi(\bmod q)$, the functional equation of $L(s, \chi)$ is

$$
\left(\frac{q}{\pi}\right)^{s / 2} \Gamma\left(\frac{s+a(\chi)}{2}\right) L(s, \chi)=\omega_{\chi}\left(\frac{q}{\pi}\right)^{(1-s) / 2} \Gamma\left(\frac{1-s+a(\chi)}{2}\right) L(1-s, \bar{\chi}),
$$

where

$$
a(\chi)=\left\{\begin{array}{ll}
0 & \text { if } \chi(-1)=1, \\
1 & \text { if } \chi(-1)=-1,
\end{array} \quad \omega_{\chi}=\frac{\tau(\chi)}{i^{a(\chi)} \sqrt{q}}\right.
$$

and

$$
\tau(\chi)=\sum_{a=1}^{q} \chi(a) e^{2 \pi i a / q} .
$$

Moreover, it is well known that a primitive character $(\bmod q)$ exists if and only if $q \not \equiv 2(\bmod 4)$; in this paper we will always assume that $q \not \equiv 2(\bmod 4)$. Hence, given $q \not \equiv 2(\bmod 4)$, the functional equation of $L(s, \chi)$ is completely determined by the signature of $\chi$, defined by

$$
s(\chi)=(a(\chi), \tau(\chi)) .
$$

Note that this is in fact a special case of a general result, obtained in the framework of the Selberg class, characterizing functional equations by the so-called basic invariants, see Kaczorowski-Perelli [10]. Indeed, for degree $1 L$-functions the basic 
invariants determining the functional equation are conductor, parity and root number (respectively $q, a(\chi)$ and $\omega_{\chi}$ in this case, but clearly one may replace $\omega_{\chi}$ by $\tau(\chi)$ ); see [9]. Our question can therefore be rephrased as follows: under what conditions two primitive characters $(\bmod q)$ with the same signature are equal? In this paper we shall determine all cases where equality of signatures implies equality of primitive characters, see Theorem 2 below.

As mentioned above, [9] contains a general converse theorem for degree $1 L$-functions in the Selberg class $\delta$, namely that $\zeta(s)$ and $L(s+i \theta, \chi)$ with $\chi$ primitive and $\theta \in \mathbb{R}$ are the only $L$-functions of degree 1 in $\delta$. Using this result and Theorem 2 below we can prove the following general version of Hamburger's theorem for Dirichlet $L$-functions. For a primitive character $\chi(\bmod q)$, let $W(\chi)$ be the set of Dirichlet series $F(s)$ satisfying the following three conditions:

(i) the coefficients $a(n)$ of $F(s)$ satisfy $a(n) \ll n^{\varepsilon}$ for every $\varepsilon>0$, and there is an integer $m$ such that $(s-1)^{m} F(s)$ is an entire function of finite order;

(ii) $\log F(s)$ is a Dirichlet series with coefficients $b(n)$ satisfying $b(n)=0$ unless $n$ is a prime power $>1$, and $b(n) \ll n^{\vartheta}$ for some $\vartheta<1 / 2$;

(iii) $F(s)$ satisfies the functional equation

$$
\left(\frac{q}{\pi}\right)^{s / 2} \Gamma\left(\frac{s+a(\chi)}{2}\right) F(s)=\omega_{\chi}\left(\frac{q}{\pi}\right)^{(1-s) / 2} \Gamma\left(\frac{1-s+a(\chi)}{2}\right) \overline{F(1-\bar{s})} .
$$

Note that clearly $L(s, \chi)$ belongs to $W(\chi)$, and that condition (ii) means that $F(s)$ is a rather general Euler product. We also denote by $\mathcal{Q}$ the set of non-negative integers $q \not \equiv 2(\bmod 4)$ of the form $q=2^{a} 3^{b} m$, with $m$ square-free and $(m, 6)=1$, and satisfying one of the following two conditions:

(a) $a \in\{0,2,3,4,5\}$ and $b \in\{0,1\}$;

(b) $a \in\{0,2,3\}$ and $b=2$.

We have

Theorem 1. If $q \in \mathbb{Q}$ then $W(\chi)=\{L(s, \chi)\}$ for every primitive character $\chi$ $(\bmod q)$ ), while if $q \notin \mathbb{Q}, q \neq \equiv 2(\bmod 4)$, there exists a primitive character $\chi$ ( $\bmod q$ ) such that $W(\chi)$ contains $L(s, \chi)$ and at least another $L(s, \psi)$ with primitive $\psi(\bmod q)$.

As remarked in [9], the above conditions defining $W(\chi)$ can be weakened, and still the same result follows. For example, the Ramanujan conjecture $a(n) \ll n^{\varepsilon}$ in (i) is not necessary, the weaker assumption that $F(s)$ is absolutely convergent for $\sigma>1$ being sufficient. Hence Theorem 1 may be expressed by saying that for $q \in \mathcal{Q}$, every primitive Dirichlet $L$-function $(\bmod q)$ is characterized by the functional equation and the multiplicativity of the coefficients. We also remark that, in view of the above 
mentioned Theorem 2 of [9], the Euler product assumption in (ii) plays an essential role in Theorem 1.

The following result is crucial for Theorem 1 and also of independent interest. Given $q \not \equiv 2(\bmod 4)$, let $s_{q}$ be the map sending each primitive character $\chi(\bmod q)$ to its signature $s(\chi)$ defined above. We have

Theorem 2. The map $s_{q}$ is injective if and only if $q \in \mathbb{Q}$.

We refer to the next section for the proof of Theorem 2 and for more general results related to it. Note that Theorem 2 immediately implies the following

Corollary. The functional equations of the $L(s, \chi)$ 's with $\chi$ primitive $(\bmod q)$ are all distinct if and only if $q \in \mathbb{Q}$.

Theorem 1 follows at once from the corollary and the above mentioned results in [9]. In fact, given a primitive character $\chi(\bmod q), W(\chi)$ is a subset of the degree 1 $L$-functions in $\delta$ and hence its elements are either $\zeta(s)$ or $L(s+i \theta, \psi)$ with $\theta \in \mathbb{R}$ and primitive $\psi$. Moreover, it is clear that $\theta=0$ and the conductor of $\psi$ is $q$ if $L(s+i \theta, \psi)$ satisfies the unshifted functional equation (1.1). Therefore, $W(\chi)$ contains only Dirichlet $L$-functions with primitive characters $(\bmod q)$ (including the trivial character $(\bmod 1))$, and hence Theorem 1 follows from the corollary.

We remark that generalizations of Hamburger's theorem to Dedekind zeta functions and to Hecke $L$-functions associated with algebraic number fields do exist in the literature, see e.g. Gurevič [6], Ehrenpreis-Kawai [5] and Yoshimoto [18]. However, although such functions include the Dirichlet $L$-functions, these results are more in the spirit of Weil's converse theorem, i.e., characterizing an $L$-function by means of analytic properties of its twits by suitable characters. In particular, as far as we can see the results of this paper do not follow from the results in the above papers. Recalling that the twist of a function $F \in W(\chi)$ by a Dirichlet character $\psi$ is by definition

$$
F(s, \psi)=\sum_{n=1}^{\infty} a(n) \psi(n) n^{-s},
$$

we state the following variant of these converse theorems, which in view of Theorem 1 is interesting only when $q \notin \mathbb{Q}$.

Theorem 3. Let $\chi_{4}$ be the odd character $(\bmod 4)$ and $\chi$ be primitive $(\bmod q)$. If $F \in W(\chi)$ satisfies $F(1)=L(1, \chi)$ if $\chi$ is even, and $F\left(1, \chi_{4}\right)=L\left(1, \chi_{4}\right)$ if $\chi$ is odd, then $F(s)=L(s, \chi)$.

The proof of Theorem 3 is a consequence of Corollary 3 in Baker-Birch-Wirsing [1], from which in particular follows that the values $L\left(1, \chi_{j}\right)$ are all distinct when $\chi_{j}$ 
runs over a finite set of even characters with distinct $\chi_{j}^{*}$, where $\chi_{j}^{*}$ denotes the primitive character inducing $\chi_{j}$. We already know that if $F \in W(\chi)$ then $F(s)=L(s, \psi)$ with $\psi$ primitive $(\bmod q)$ having the same signature of $\chi$, hence the case of even $\chi$ follows at once. If $\chi$ is odd then $\psi \chi_{4}$ and $\chi \chi_{4}$ are even characters $\left(\bmod q^{\prime}\right)$ for a certain $q^{\prime} \mid 4 q$, and hence $\left(\psi \chi_{4}\right)^{*}=\left(\chi_{4}\right)^{*}$. Let $(a, q)=1$ and let $p \equiv a(\bmod q)$ be an odd prime, so that $\left(p, q^{\prime}\right)=1$. By definition of induced character we have

$$
\begin{aligned}
\psi(a) & =\psi(p)=\chi_{4}(p)^{-1}\left(\psi \chi_{4}\right)(p)=\chi_{4}(p)^{-1}\left(\psi \chi_{4}\right)^{*}(p) \\
& =\chi_{4}(p)^{-1}\left(\chi_{4}\right)^{*}(p)=\chi_{4}(p)^{-1} \chi \chi_{4}(p)=\chi(p)=\chi(a),
\end{aligned}
$$

thus $\psi=\chi$ and Theorem 3 follows in this case as well.

We point out the interesting problem of determining the cardinality $|W(\chi)|$ of $W(\chi)$ for $q \notin \mathcal{Q}$, the trivial upper bound being of course

$$
|W(\chi)| \leq \varphi^{*}(q)=\prod_{p^{k} \| q}\left(\varphi\left(p^{k}\right)-\varphi\left(p^{k-1}\right)\right),
$$

the number of primitive characters $(\bmod q)$. Note that the problem asks, in particular, for a characterization of the primitive $\chi(\bmod q)$ such that $W(\chi)=\{L(s, \chi)\}$ when $q \notin \mathcal{Q}$. This problem has been recently studied by Molteni [12], [13].

We conclude with a numerical example of distinct Dirichlet $L$-functions satisfying the same functional equation. We use the standard notation $e(x)=e^{2 \pi i x}$, and let $\zeta_{q}=e(1 / q)$. Moreover, given a character $\chi(\bmod q)$ we denote by $\chi(\mathbb{Z})$ the set of values taken by $\chi$ over the integers and write $K_{\chi}=\mathbb{Q}\left(\zeta_{q}, \chi(\mathbb{Z})\right)$ and $k_{\chi}=$ $\mathbb{Q}\left(\zeta_{q}, \tau(\chi)\right)$, so that $K_{\chi} / k_{\chi}$ is a field extension. Choose $q=25$ and note that $\varphi(25)=20$ and that $\mathbb{Z}_{25}^{*}$ is generated by 2 . We consider the primitive character $\chi$ $(\bmod 25)$ defined by $\chi(2)=\zeta_{20}^{7}$. Then we have

$$
\tau(\chi)=\sum_{j=0}^{19} \chi\left(2^{j}\right) \zeta_{25}^{2^{j}}=\sum_{j=0}^{19} e\left(\frac{35 j+2^{j+2}}{100}\right),
$$

and a computation shows that $\tau(\chi)=5 \zeta_{25}$. Hence in this case $k_{\chi}=\mathbb{Q}\left(\zeta_{25}\right)$ while $K_{\chi}=\mathbb{Q}\left(\zeta_{25}, \zeta_{20}\right)=\mathbb{Q}\left(\zeta_{100}\right)$, thus $k_{\chi} \neq K_{\chi}$; in fact $\left[K_{\chi}: k_{\chi}\right]=2$. Therefore, there exists a unique non-trivial automorphism $\sigma \in \operatorname{Gal}\left(K_{\chi} / k_{\chi}\right)$, and we define $\chi^{\sigma}(a)=\sigma(\chi(a))$, which is clearly a primitive character $(\bmod q)$ with the same parity of $\chi$. Moreover, since $\sigma$ fixes $k_{\chi}$ we have

$$
\tau(\chi)=\sigma(\tau(\chi))=\sum_{a=1}^{q} \chi^{\sigma}(a) \zeta_{q}^{a}=\tau\left(\chi^{\sigma}\right) .
$$

Finally, $\chi^{\sigma} \neq \chi$ since otherwise $\sigma(\chi(a))=\chi(a)$ for all $a \in \mathbb{Z}$ and hence $\sigma$ would fix $K_{\chi}$, a contradiction; in particular, one finds that $\chi^{\sigma}(2)=\zeta_{20}^{17}$. Therefore, $L(s, \chi)$ and $L\left(s, \chi^{\sigma}\right)$ are distinct but satisfy the same functional equation. 
Acknowledgments. We wish to warmly thank Umberto Zannier, who read a previous version of this manuscript and suggested substantial improvements. This research was partially supported by a MUR grant Cofin2006 and by the grant N N201 148233 from the Polish Ministry of Science and Higher Education.

\section{Proof of Theorem 2}

We first recall some basic properties of the Gauss sums and of the groups $\mathbb{Z}_{p^{k}}^{*}$. In general, given a character $\chi(\bmod q)$ and a primitive $q$-th root of unity $\zeta_{q}$, the associated Gauss sum is

$$
\tau\left(\chi, \zeta_{q}\right)=\sum_{c=1}^{q} \chi(c) \zeta_{q}^{c}
$$

and depends on $\zeta_{q}$ according to the relation

$$
\chi(e) \tau\left(\chi, \zeta_{q}^{e}\right)=\tau\left(\chi, \zeta_{q}\right), \quad(e, q)=1 .
$$

The classical Gauss sum $\tau(\chi)$ corresponds to the choice $\zeta_{q}=e(1 / q)$. Suppose that $\left(q_{1}, q_{2}\right)=1, \chi_{i}$ are primitive characters $\left(\bmod q_{i}\right)$ and let $\zeta_{q_{i}}$ be primitive $q_{i}$-th roots, $i=1,2$. Then $\chi_{1} \chi_{2}$ is a primitive character $\left(\bmod q_{1} q_{2}\right), \zeta_{q_{1}} \zeta_{q_{2}}$ is a primitive $q_{1} q_{2}$-th root and

$$
\tau\left(\chi_{1} \chi_{2}, \zeta_{q_{1}} \zeta_{q_{2}}\right)=\tau\left(\chi_{1}, \zeta_{q_{1}}\right) \tau\left(\chi_{2}, \zeta_{q_{2}}\right)
$$

and viceversa. Note that (2.1) shows the 2-variables multiplicativity of the general Gauss sums. When dealing with Gauss sums $\tau\left(\chi, \zeta_{q}\right)$, the signature of $\chi$ is $\left(a(\chi), \tau\left(\chi, \zeta_{q}\right)\right)$ and is denoted by $s\left(\chi, \zeta_{q}\right)$.

Explicit formulae for Gauss sums modulo prime-powers were given by Odoni [14] and Mauclaire [11], see also Chapter 1 of Berndt-Evans-Williams [2]. These formulae can easily be used to compute the value of Gauss sums modulo $p^{k}$ for each fixed $k$ and every fixed character, and in fact we used them to compute Tables 1-5 below. ${ }^{1}$ However, such formulae contain products of several roots of unity whose dependence on the character is quite involved, and it is not clear how to use them directly to prove our results.

The structure of the groups $\mathbb{Z}_{p^{k}}^{*}$ for $p$ odd prime and $p=2$ is quite different. Let $p$ be an odd prime. The group $\mathbb{Z}_{p^{k}}^{*}$ is cyclic of order $(p-1) p^{k-1}$. Let $U_{k}=$ $\left\{x \in \mathbb{Z}_{p^{k}}^{*}: x^{p-1}=1\right\}$ and $V_{k}=\left\{x \in \mathbb{Z}_{p^{k}}^{*}: x^{p^{k-1}}=1\right\}$. Then $\left|U_{k}\right|=p-1$, $\left|V_{k}\right|=p^{k-1}, \mathbb{Z}_{p^{k}}^{*}$ is the direct product of $U_{k}$ and $V_{k}$ and if $g$ is a generator of $\mathbb{Z}_{p^{k}}^{*}$, then $g^{p^{k-1}}$ generates $U_{k}$ and $g^{p-1}$ generates $V_{k}$. The map $U_{k} \rightarrow \mathbb{Z}_{p}^{*}$ sending $x$ to its congruence class $(\bmod p)$ provides an isomorphism of groups so that for every

\footnotetext{
${ }^{1}$ The values of the Gauss sums are normalized dividing by the square-root of the conductor. In the tables we use the notation $\zeta_{q}=e(1 / q)$. For every root, some characters having the same Gauss sum are shown in bold.
} 


\begin{tabular}{c|c|c|c|c|} 
Char. $\backslash$ root & $\zeta_{8}$ & $\zeta_{8}^{3}$ & $\zeta_{8}^{5}$ & $\zeta_{8}^{7}$ \\
\cline { 1 - 2 }$\chi(-1)=1, \quad \chi(5)=-1$ & 1 & -1 & -1 & 1 \\
$\chi(-1)=-1, \chi(5)=-1$ & $i$ & $i$ & $-i$ & $-i$ \\
\hline
\end{tabular}

Table 1. Gauss sums for primitive characters $\left(\bmod 2^{3}\right)$ and primitive $2^{3}$-th roots of unity.

\begin{tabular}{|c|c|c|c|c|c|c|c|c|}
\hline Char. \root & $\zeta_{16}$ & $\zeta_{16}^{3}$ & $\zeta_{16}^{5}$ & $\zeta_{16}^{7}$ & $\zeta_{16}^{9}$ & $\zeta_{16}^{11}$ & $\zeta_{16}^{13}$ & $\zeta_{16}^{15}$ \\
\hline $\begin{array}{c}\chi(-1)=1 \\
\chi(5)=i\end{array}$ & $\zeta_{16}^{-1}$ & $\zeta_{16}^{3}$ & $\zeta_{16}^{-5}$ & $\zeta_{16}^{7}$ & $\zeta_{16}^{7}$ & $\zeta_{16}^{-5}$ & $\zeta_{16}^{3}$ & $\zeta_{16}^{-1}$ \\
\hline$\chi(5)=-i$ & $\zeta_{16}$ & $\zeta_{16}^{-3}$ & $\zeta_{16}^{5}$ & $\zeta_{16}^{-7}$ & $\zeta_{16}^{-7}$ & $\zeta_{16}^{5}$ & $\zeta_{16}^{-3}$ & $\zeta_{16}$ \\
\hline $\begin{array}{c}\chi(-1)=-1 \\
\chi(5)=i\end{array}$ & $\zeta_{16}^{7}$ & $\zeta_{16}^{3}$ & $\zeta_{16}^{3}$ & $\zeta_{16}^{7}$ & $\zeta_{16}^{-1}$ & $\zeta_{16}^{-5}$ & $\zeta_{16}^{-5}$ & $\zeta_{16}^{-1}$ \\
\hline$\chi(5)=-i$ & $\zeta_{16}$ & $\zeta_{16}^{5}$ & $\zeta_{16}^{5}$ & $\zeta_{16}$ & $\zeta_{16}^{-7}$ & $\zeta_{16}^{-3}$ & $\zeta_{16}^{-3}$ & $\zeta_{16}^{-7}$ \\
\hline
\end{tabular}

Table 2. Gauss sums for primitive characters $\left(\bmod 2^{4}\right)$ and primitive $2^{4}$-th roots of unity.

integer $z$, the equation $x=z(\bmod p)$ has a (unique) solution $x \in U_{k}$ if and only if $p \nmid z$. Given a generator $g$ of $\mathbb{Z}_{p^{k}}^{*}$, the characters $\chi\left(\bmod p^{k}\right)$ are determined by the integer $\alpha_{\chi}$, unique $\left(\bmod \varphi\left(p^{k}\right)\right)$, such that

$$
\chi(g)=e\left(\alpha_{\chi} / \varphi\left(p^{k}\right)\right)
$$

Moreover, $\chi$ is even if and only if $\alpha_{\chi}$ is even and is primitive if and only if $p \nmid \alpha_{\chi}$. The decomposition $\mathbb{Z}_{p^{k}}^{*}=U_{k} \times V_{k}$ corresponds to a decomposition of each character $\chi\left(\bmod p^{k}\right)$ as $\chi=\chi_{U} \chi_{V}$, where $\chi_{U}\left(\right.$ resp. $\left.\chi_{V}\right)$ is a character of $U_{k}$ (resp. of $\left.V_{k}\right)$, i.e., a homomorphism from $U_{k}$ (resp. $V_{k}$ ) to $\mathbb{C}^{*}$. According to this decomposition, $\chi$ is primitive if and only if among the values of $\chi_{V}$ there are primitive $p^{k-1}$-th roots of unity, and in this case $\chi_{V}$ is called primitive. Moreover, equality $-1=g^{p^{k-1}(p-1) / 2}$ shows that $-1 \in U_{k}$ so that $\chi$ is even if and only if $\chi_{U}(-1)=1$.

Let $p=2$. When $k>2$ group $\mathbb{Z}_{2^{k}}^{*}$ is isomorphic to the direct product of a cyclic group of order 2 and a cyclic group of order $2^{k-2}$, respectively generated by 


\begin{tabular}{|c|c|c|c|c|c|c|c|c|}
\hline Char. \root & $\zeta_{32}$ & $\zeta_{32}^{3}$ & $\zeta_{32}^{5}$ & $\zeta_{32}^{7}$ & $\zeta_{32}^{9}$ & $\zeta_{32}^{11}$ & $\zeta_{32}^{13}$ & $\zeta_{32}^{15}$ \\
\hline $\begin{aligned} \chi(-1) & =1 \\
\chi(5) & =\zeta_{8}\end{aligned}$ & $\zeta_{32}^{5}$ & $\zeta_{32}^{-7}$ & $\zeta_{32}$ & $\zeta_{32}^{-3}$ & $\zeta_{32}^{13}$ & $\zeta_{32}^{-15}$ & $\zeta_{32}^{9}$ & $\zeta_{32}^{-11}$ \\
\hline$\chi(5)=\zeta_{8}^{3}$ & $\zeta_{32}^{3}$ & $\zeta_{32}^{-1}$ & $\zeta_{32}^{-9}$ & $\zeta_{32}^{11}$ & $\zeta_{32}^{-5}$ & $\zeta_{32}^{7}$ & $\zeta_{32}^{15}$ & $\zeta_{32}^{-13}$ \\
\hline$\chi(5)=\zeta_{8}^{5}$ & $\zeta_{32}^{-3}$ & $\zeta_{32}$ & $\zeta_{32}^{9}$ & $\zeta_{32}^{-11}$ & $\zeta_{32}^{5}$ & $\zeta_{32}^{-7}$ & $\zeta_{32}^{-15}$ & $\zeta_{32}^{13}$ \\
\hline$\chi(5)=\zeta_{8}^{7}$ & $\zeta_{32}^{-5}$ & $\zeta_{32}^{7}$ & $\zeta_{32}^{-1}$ & $\zeta_{32}^{3}$ & $\zeta_{32}^{-13}$ & $\zeta_{32}^{15}$ & $\zeta_{32}^{-9}$ & $\zeta_{32}^{11}$ \\
\hline $\begin{aligned} \chi(-1) & =-1 \\
\chi(5) & =\zeta_{8}\end{aligned}$ & $\zeta_{32}^{5}$ & $\zeta_{32}^{9}$ & $\zeta_{32}$ & $\zeta_{32}^{13}$ & $\zeta_{32}^{13}$ & $\zeta_{32}$ & $\zeta_{32}^{9}$ & $\zeta_{32}^{5}$ \\
\hline$\chi(5)=\zeta_{8}^{3}$ & $\zeta_{32}^{-13}$ & $\zeta_{32}^{-1}$ & $\zeta_{32}^{7}$ & $\zeta_{32}^{11}$ & $\zeta_{32}^{11}$ & $\zeta_{32}^{7}$ & $\zeta_{32}^{-1}$ & $\zeta_{32}^{-13}$ \\
\hline$\chi(5)=\zeta_{8}^{5}$ & $\zeta_{32}^{-3}$ & $\zeta_{32}^{-15}$ & $\zeta_{32}^{9}$ & $\zeta_{32}^{5}$ & $\zeta_{32}^{5}$ & $\zeta_{32}^{9}$ & $\zeta_{32}^{-15}$ & $\zeta_{32}^{-3}$ \\
\hline$\chi(5)=\zeta_{8}^{7}$ & $\zeta_{32}^{11}$ & $\zeta_{32}^{7}$ & $\zeta_{32}^{15}$ & $\zeta_{32}^{3}$ & $\zeta_{32}^{3}$ & $\zeta_{32}^{15}$ & $\zeta_{32}^{7}$ & $\zeta_{32}^{11}$ \\
\hline
\end{tabular}

Table 3. Gauss sums for primitive characters $\left(\bmod 2^{5}\right)$ and half of the primitive $2^{5}$-th roots of unity. The values for the roots $\zeta_{q}^{a}$ with $a>15$ can be deduced using the identity $\tau\left(\chi, \bar{\zeta}_{q}^{a}\right)=$ $\chi(-1) \tau\left(\chi, \zeta_{q}^{a}\right)$.

-1 and 5. It follows that the characters $\left(\bmod 2^{k}\right)$ are uniquely determined by a couple of integers $(\alpha, \beta)$ with $\alpha(\bmod 2)$ and $\beta\left(\bmod 2^{k-2}\right)$ such that

$$
\chi_{\alpha, \beta}(-1)=(-1)^{\alpha}, \quad \chi_{\alpha, \beta}(5)=e\left(\beta / 2^{k-2}\right),
$$

and $\chi_{\alpha, \beta}$ is even if and only if $\alpha$ is even and is primitive if and only if $\beta$ is odd.

We recall that $q \in \mathcal{Q}$ if and only if $q=2^{a} 3^{b} m$ with $(m, 6)=1, m$ square-free and one of conditions (a) and (b) in the introduction is satisfied. Let $\zeta_{q}$ be a $q$-th primitive root of unity and denote by $s_{q, \zeta_{q}}$ the map sending primitive $\chi$ 's $(\bmod q)$ to $s\left(\chi, \zeta_{q}\right)$. We start with

Proposition 1. Let $q \in \mathbb{Q}$ and let $\zeta_{q}$ be a $q$-th primitive root of unity. Then the map $s_{q, \zeta_{q}}$ is injective.

Proof. Let $q \in \mathcal{Q}, q=2^{a} 3^{b} m$, suppose that $m>1$ and let $p$ be the largest prime factor of $m$. Writing $q^{\prime}=q / p$, clearly $p$ and $q^{\prime}$ are coprime. Given a primitive $\chi$ 
$(\bmod q)$ and a primitive $q$-th $\operatorname{root} \zeta_{q}$, let $\chi=\chi^{\prime} \chi_{p}$ be the decomposition of $\chi(\bmod q)$ as a product of $\chi^{\prime}\left(\bmod q^{\prime}\right)$ and $\chi_{p}(\bmod p)$. Moreover, thanks to (2.1), let $\zeta_{q^{\prime}}$ and $\zeta_{p}$ be the primitive $q^{\prime}$-th and $p$-th roots satisfying

$$
\tau\left(\chi, \zeta_{q}\right)=\tau\left(\chi^{\prime}, \zeta_{q^{\prime}}\right) \tau\left(\chi_{p}, \zeta_{p}\right)
$$

Consider the two cyclotomic fields $K_{\chi}^{0}=\mathbb{Q}\left(\zeta_{\varphi(q)}, \zeta_{q^{\prime}}\right)$ and $K_{\chi}=K_{\chi}^{0}\left(\zeta_{p}\right)$, where $\zeta_{\varphi(q)}=e(1 / \varphi(q))$. Since $p$ is the largest prime dividing $m$, we have that $\left(p, q^{\prime} \varphi(q)\right)=1$, and hence $\left[K_{\chi}: K_{\chi}^{0}\right]=\varphi(p)$. Therefore, by elementary Galois theory, for every $(a, p)=1$ there exists an automorphism $\sigma_{a} \in \operatorname{Gal}\left(K_{\chi} / K_{\chi}^{0}\right)$ such that $\sigma_{a}\left(\zeta_{p}\right)=\zeta_{p}^{a}$. Since $\sigma_{a}$ fixes $\zeta_{\varphi(q)}$, it fixes also the values of $\chi, \chi^{\prime}$ and $\chi_{p}$. Moreover, $\sigma_{a}$ fixes also $\zeta_{q^{\prime}}$, hence by (2.2) we have

$$
\begin{aligned}
\sigma_{a}\left(\tau\left(\chi, \zeta_{q}\right)\right) & =\sigma_{a}\left(\tau\left(\chi^{\prime}, \zeta_{q^{\prime}}\right) \tau\left(\chi_{p}, \zeta_{p}\right)\right)=\tau\left(\chi^{\prime}, \zeta_{q^{\prime}}\right) \sigma_{a}\left(\tau\left(\chi_{p}, \zeta_{p}\right)\right) \\
& =\tau\left(\chi^{\prime}, \zeta_{q^{\prime}}\right) \tau\left(\chi_{p}, \zeta_{p}^{a}\right)=\tau\left(\chi^{\prime}, \zeta_{q^{\prime}}\right) \overline{\chi_{p}(a)} \tau\left(\chi_{p}, \zeta_{p}\right) \\
& =\overline{\chi_{p}(a)} \tau\left(\chi, \zeta_{q}\right),
\end{aligned}
$$

thus for every $(a, p)=1$

$$
\chi_{p}(a)=\tau\left(\chi, \zeta_{q}\right) / \sigma_{a}\left(\tau\left(\chi, \zeta_{q}\right)\right)
$$

Hence $\chi_{p}$ is completely determined by the value of $\tau\left(\chi, \zeta_{q}\right)$. In particular, if $\chi, \psi$ are primitive characters $(\bmod q)$ with $\tau\left(\chi, \zeta_{q}\right)=\tau\left(\psi, \zeta_{q}\right)$ then, with the above notation, $\chi_{p}=\psi_{p}$ and by (2.2) also

$$
\tau\left(\chi^{\prime}, \zeta_{q^{\prime}}\right)=\tau\left(\psi^{\prime}, \zeta_{q^{\prime}}\right)
$$

Iterating the argument we eliminate all factors of $m$, thus we may assume that $m=1$.

Suppose now that $q=2^{a} 3^{b}$ with $(a, b) \neq(0,0)$ and that we are in case (a), i.e., $a \in\{0,2,3,4,5\}$ and $b \in\{0,1\}$. If $b=1$ we have $\varphi^{*}(3)=1$, see (1.2), hence by the previous argument we eliminate the factor 3 , so we may assume that $q=2^{a}$ with $a \in\{0,2,3,4,5\}$. If $a=2$ we have $\varphi^{*}(4)=1$, while in the other cases the result follows by direct computation of the Gauss sums, see Tables 1, 2 and 3. In case (b), i.e., $a \in\{0,2,3\}$ and $b=2$, the result follows again by direct computation of the Gauss sums for $q=2^{a} 3^{2}$, see Tables 1 and 5. Proposition 1 is therefore proved.

Now we deal with the case $q=p^{k}$, where $p$ is an odd prime and $k \geq 2$ (if $p=3$ then $k \geq 3$ ), and prove the following

Proposition 2. Let $q=p^{k}$ with a prime $p \geq 5$ and $k \geq 2$ or $p=3$ and $k \geq 3$, and let $\zeta_{q}$ be a primitive $q$-th root of unity. Then there exist two distinct primitive characters $\chi, \psi(\bmod q)$ with $s\left(\chi, \zeta_{q}\right)=s\left(\psi, \zeta_{q}\right)$. 


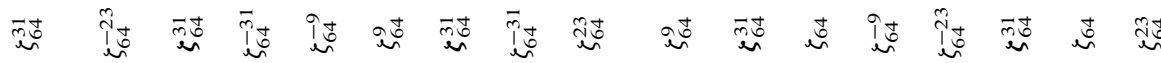

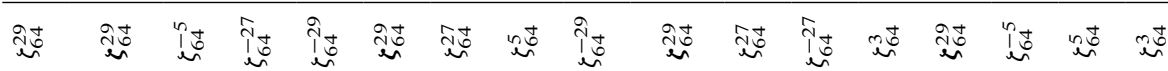

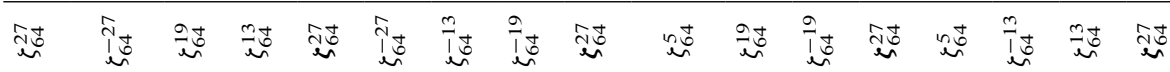

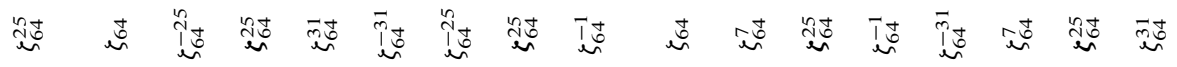

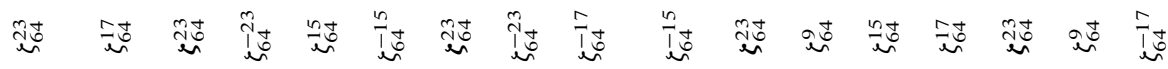

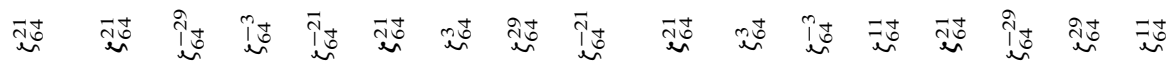

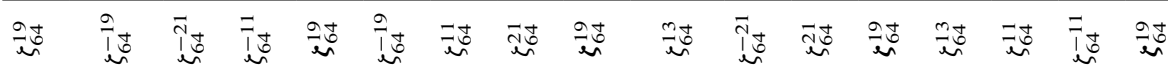

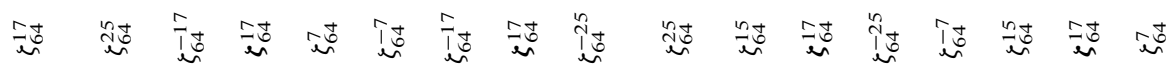

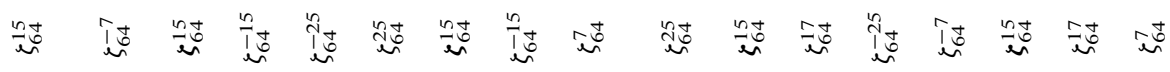

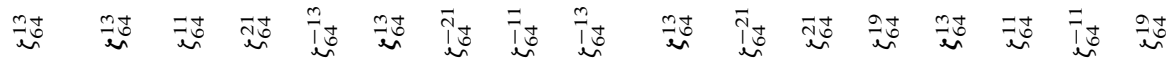

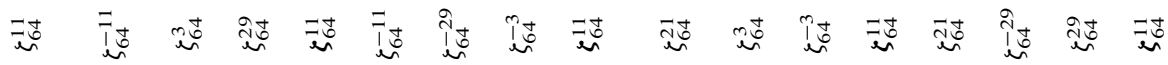

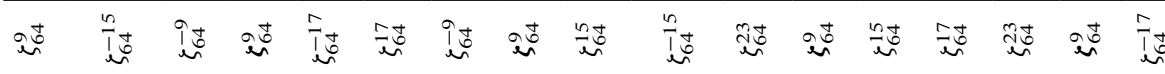

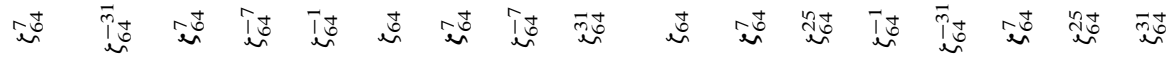

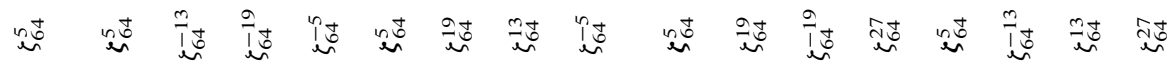

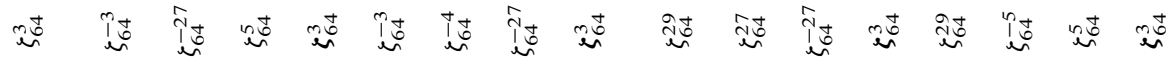

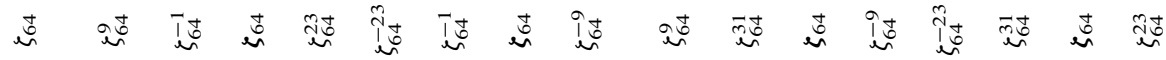

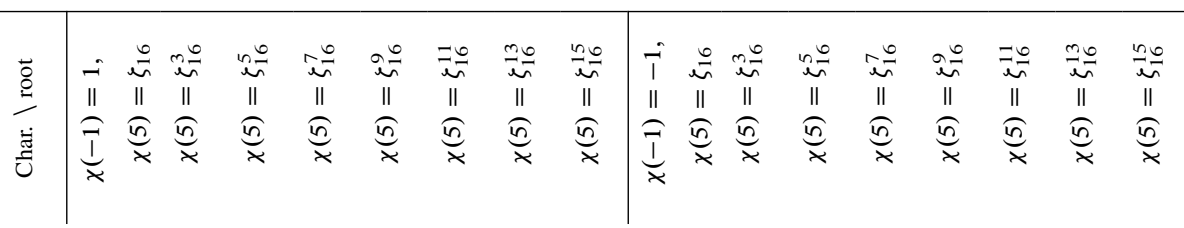

Table 4. Gauss sums for primitive characters $\left(\bmod 2^{6}\right)$ and half of the primitive $2^{6}$-th roots of unity. The values for the roots $\zeta_{q}^{a}$ with $a>31$ can be deduced using the identity $\tau\left(\chi, \bar{\zeta}_{q}^{a}\right)=$ $\chi(-1) \tau\left(\chi, \zeta_{q}^{a}\right)$. 


\begin{tabular}{c|c|c|c|c|c|c|} 
Char. \root & $\zeta_{9}$ & $\zeta_{9}^{2}$ & $\zeta_{9}^{4}$ & $\zeta_{9}^{5}$ & $\zeta_{9}^{7}$ & $\zeta_{9}^{8}$ \\
\cline { 1 - 1 }$\chi(2)=\zeta_{6}$ & $-\zeta_{9}^{8}$ & $\zeta_{9}^{2}$ & $-\zeta_{9}^{7}$ & $\zeta_{9}^{5}$ & $-\zeta_{9}^{2}$ & $\zeta_{9}^{8}$ \\
$\chi(2)=\zeta_{6}^{2}$ & $\zeta_{9}$ & $\zeta_{9}^{7}$ & $\zeta_{9}^{4}$ & $\zeta_{9}^{4}$ & $\zeta_{9}^{7}$ & $\zeta_{9}$ \\
$\chi(2)=\zeta_{6}^{4}$ & $\zeta_{9}^{8}$ & $\zeta_{9}^{2}$ & $\zeta_{9}^{5}$ & $\zeta_{9}^{5}$ & $\zeta_{9}^{2}$ & $\zeta_{9}^{8}$ \\
$\chi(2)=\zeta_{6}^{5}$ & $\zeta_{9}$ & $-\zeta_{9}^{7}$ & $\zeta_{9}^{4}$ & $-\zeta_{9}^{4}$ & $\zeta_{9}^{7}$ & $\zeta_{9}^{2}$ \\
\hline
\end{tabular}

Table 5. Gauss sums for primitive characters $\left(\bmod 3^{2}\right)$ and the primitive $3^{2}$-th roots of unity.

Note that the primitive characters (mod 9) have distinct signatures with respect to every choice for the 9-th root of unity (see Table 5). In the proof of Proposition 2 we need Lemma 1 below. Let $q$ and $\zeta_{q}$ be as in Proposition 2 and $\chi$ be a character $(\bmod q)$. The decomposition $\mathbb{Z}_{q}^{*}=U_{k} \times V_{k}$ described at the beginning of this section allows one to write

$$
\tau\left(\chi, \zeta_{q}\right)=\sum_{c} \chi(c) \zeta_{q}^{c}=\sum_{\substack{u \in U_{k} \\ v \in V_{k}}} \chi(u v) \zeta_{q}^{u v}=\sum_{u \in U_{k}} \chi(u) \zeta_{q}^{u} T(\chi, u)
$$

where

$$
T(\chi, u)=\sum_{v \in V_{k}} \chi(v) \zeta_{q}^{u(v-1)}
$$

Let $g$ be a generator of $\mathbb{Z}_{q}^{*}$ and $w(\bmod p)$ be such that

$$
g^{p-1}=1+w p\left(\bmod p^{2}\right) .
$$

Since $g^{p-1}$ generates $V_{k}$ whose order is $p^{k-1}, \chi\left(g^{p-1}\right)$ is a $p^{k-1}$-th roots of unity. Hence $\chi\left(g^{p-1}\right)=\zeta_{q}^{\gamma p}$ for some uniquely determined $\gamma\left(\bmod p^{k-1}\right)$; moreover, $(\gamma, p)=1$ if and only if $\chi$ is primitive. Therefore, the congruence

$$
u w \equiv-\gamma(\bmod p)
$$

has a solution $u \in U_{k}$ only when $\gamma \not \equiv 0(\bmod p)$, i.e., only for primitive characters, and in this case it is unique and is denoted by $u_{\chi}$. With such a notation we have

Lemma 1. Let $q$ and $\zeta_{q}$ be as in Proposition 2 and $\chi$ be a primitive character $(\bmod q)$. Then

$$
\tau\left(\chi, \zeta_{q}\right)=\chi\left(u_{\chi}\right) \zeta_{q}^{u_{\chi}} T\left(\chi, u_{\chi}\right)
$$


Proof. Let $u$ satisfy $u w \not \equiv-\gamma(\bmod p)$. Since $V_{k}$ is generated by $g_{p}=g^{p-1}$, writing $f_{u}(h)=\gamma h p+u\left(g_{p}^{h}-1\right)(\bmod q)$ we have

$$
\begin{aligned}
T(\chi, u) & =\sum_{v \in V_{k}} \chi(v) \zeta_{q}^{u(v-1)}=\sum_{h=1}^{p^{k-1}} \chi\left(g_{p}^{h}\right) \zeta_{q}^{u\left(g_{p}^{h}-1\right)} \\
& =\sum_{h=1}^{p^{k-1}} \zeta_{q}^{\gamma h p} \zeta_{q}^{u\left(g_{p}^{h}-1\right)}=\sum_{h=1}^{p^{k-1}} \zeta_{q}^{f_{u}(h)} .
\end{aligned}
$$

Suppose that $f_{u}\left(h_{1}\right)=f_{u}\left(h_{2}\right)$ with $h_{1} \neq h_{2}$, and let $h_{1}-h_{2}=p^{v} l$ with some $v$ and $(l, p)=1$. We write $f_{u}\left(h_{1}\right) \equiv f_{u}\left(h_{2}\right)(\bmod q)$ as

$$
\gamma\left(h_{1}-h_{2}\right) p+u g_{p}^{h_{2}}\left(g_{p}^{h_{1}-h_{2}}-1\right) \equiv 0(\bmod q) .
$$

By induction on $v$ we get

$$
g_{p}^{l p^{v}} \equiv 1+l w p^{v+1}\left(\bmod p^{v+2}\right) .
$$

The conditions $1 \leq h_{1}, h_{2} \leq p^{k-1}$ imply that $v+2 \leq k$, hence we may consider (2.6) as a congruence $\left(\bmod p^{v+2}\right)$. Inserting (2.7) in (2.6) we get

$$
\gamma l p^{v+1}+u g_{p}^{h_{2}} l w p^{v+1} \equiv 0\left(\bmod p^{v+2}\right)
$$

so that

$$
\gamma l+u g_{p}^{h_{2}} l w \equiv 0(\bmod p),
$$

and since $(l, p)=1$ and $g_{p} \equiv 1(\bmod p)$ we have $\gamma+u w \equiv 0(\bmod p)$, a contradiction. Therefore, for every $u$ with $u w \neq \equiv-\gamma(\bmod p)$ the map $f_{u}: \mathbb{Z}_{p^{k-1}} \rightarrow$ $\mathbb{Z}_{p^{k}}$ is injective. Since $p \mid f_{u}(h)$ for every $h$, it follows that the values $f_{u}(h)$ run over a complete set of representatives of $p \mathbb{Z}_{p^{k-1}}$ as $h$ runs over $\mathbb{Z}_{p^{k-1}}$. Thus by (2.5)

$$
T(\chi, u)=\sum_{h=1}^{p^{k-1}} \zeta_{q}^{f_{u}(h)}=\sum_{h=1}^{p^{k-1}} \zeta_{q}^{p h}=0
$$

for every $u$ such that $u w \not \equiv-\gamma(\bmod p)$, and the lemma follows from (2.3).

Let $\chi=\chi_{U} \chi_{V}$ be the decomposition reported at the beginning of this section. Note that $u_{\chi}$ and $T\left(\chi, u_{\chi}\right)$ depend only on the values assumed by $\chi$ on $V_{k}$, i.e., depend only on $\chi_{V}$. In order to get distinct primitive characters with the same signature we make use of the following strategy. We fix a primitive character $\eta$ of $V_{k}$ and consider the $p-1$ distinct primitive characters $(\bmod q)$ of the form $\chi_{U} \eta$, where $\chi_{U}$ varies 
over the characters of $U_{k}$. Hence if $\chi$ and $\psi$ are two such characters, $\zeta_{q}$ is a $q$-th primitive root and $\tilde{u}=u_{\chi}=u_{\psi}$, by Lemma 1 we have

$$
\frac{\tau\left(\chi_{U} \eta, \zeta_{q}\right)}{\tau\left(\psi_{U} \eta, \zeta_{q}\right)}=\frac{\chi_{U}(\tilde{u})}{\psi_{U}(\tilde{u})}
$$

Since $U_{k}$ is cyclic, there exist $\chi_{U} \neq \psi_{U}$ with $\chi_{U}(\tilde{u})=\psi_{U}(\tilde{u})$ if and only if $\tilde{u}$ is not a generator of $U_{k}$. The best choice for $\eta$ would be a primitive character for which $\tilde{u}=1$, since in this case (2.8) shows that $\tau\left(\chi, \zeta_{q}\right)=\tau\left(\psi, \zeta_{q}\right)$, i.e., there are $p-1$ distinct primitive characters $(\bmod q)$ with the same Gauss sum. Moreover, both a couple of even and a couple of odd characters are produced whenever $p-1 \geq 4$. For $p=3$ this approach produces only $p-1=2$ characters having necessarily different parity. As a consequence, the case $p=3$ must be treated with an ad hoc argument. The following proof of Proposition 2 shows, in particular, that indeed a character $\eta$ with $\tilde{u}=1$ can always be found.

Proof of Proposition 2. We look for characters $\chi$ having $u_{\chi}=1$, and (2.4) shows that $u_{\chi}=1$ if and only if $\gamma \equiv-w(\bmod p)$. The identity $\chi\left(g^{p-1}\right)=\zeta_{q}^{\gamma p}$ implies that the integer $\alpha_{\chi}$ introduced at the beginning of this section satisfies $\alpha_{\chi} \equiv c \gamma$ (mod $p^{k-1}$ ) where $c$ is such that $\zeta_{q}=e(c / q)$, thus $u_{\chi}=1$ if and only if $\alpha_{\chi} \equiv-c w$ $(\bmod p)$. As a consequence, there are $\varphi\left(p^{k-1}\right)$ distinct characters $\chi$ having $u_{\chi}=1$, corresponding to the choices

$$
\alpha_{\chi}=-c w+h p, \quad h=1, \ldots, \varphi\left(p^{k-1}\right) .
$$

Among such characters $\chi$, those having the same $\gamma$ (and hence equal on $V_{k}$ ) must satisfy

$$
\alpha_{\chi}=-c w+h p^{k-1}, \quad h=1, \ldots, p-1 .
$$

In this way we produce $p-1$ distinct primitive characters $(\bmod q)$ all having $\tilde{u}=1$ and hence the same Gauss sum thanks to (2.8). Therefore, if $p \geq 5$ and $k \geq 2$ this argument produces at least two distinct primitive characters $\left(\bmod p^{k}\right)$ with the same signature (in fact, it gives a bit more, as mentioned above).

Note that if $p=3$ and $k \geq 2$ the previous argument produces only two distinct characters, with different parity since in this case $h$ assumes only values 1 and 2 . In order to complete the proof we show that if $p=3$ and $k \geq 3$ there exist two primitive characters having distinct $\gamma$ but with the same Gauss sum and parity; we explicitly construct such a couple of characters. We first observe that

$$
4^{2 \cdot 3^{k-3}} \equiv 1+5 \cdot 3^{k-2}\left(\bmod 3^{k}\right) \quad \text { for all } k \geq 3,
$$

which can be easily verified by induction on $k$. Next we set

$$
f_{0}(l)=6 l+4^{l}-1, \quad f_{1}(l)=6 l\left(1+3^{k-2}\right)+4^{l}-1
$$


and prove that, writing $l_{0}=2 \cdot 3^{k-3}$, for every $l$ and every $k \geq 3$ we have

$$
f_{1}(l) \equiv f_{0}\left(l+l_{0}\right)\left(\bmod 3^{k}\right) .
$$

The proof is simple, since $\left(\bmod 3^{k}\right)$ we have

$$
\begin{aligned}
f_{0}\left(l+l_{0}\right)-f_{1}(l) & =6\left(l+l_{0}\right)+4^{l+l_{0}}-1-\left(6 l\left(1+3^{k-2}\right)+4^{l}-1\right) \\
& =4^{l+l_{0}}-4^{l}-2 l 3^{k-1}+6 l_{0} \\
& =4^{l}\left(4^{l_{0}}-1\right)-2 l 3^{k-1}+6 l_{0} \\
& \equiv 4^{l} 5 \cdot 3^{k-2}-2 l 3^{k-1}+4 \cdot 3^{k-2}=3^{k-2}\left(4^{l} 5-6 l+4\right),
\end{aligned}
$$

and $4^{l} 5-6 l+4 \equiv 0(\bmod 9)$ since it holds for $l=0,1,2$ and the left hand side is periodic with period 3 when considered $(\bmod 9)$. Now we choose $g=2$ as generator of $\mathbb{Z}_{3^{k}}^{*}$, so $g^{p-1}=1+3$ and hence $w=1$. We show that the characters $\chi$ with $\alpha_{\chi}=2$ and $\psi$ with $\alpha_{\psi}=2+2 \cdot 3^{k-2}$ have the same Gauss sum; note that both are even primitive characters. For both characters $\alpha_{\chi}=\alpha_{\psi}=-1$ (mod 3) (since $k \geq 3$ ), so $u_{\chi}=u_{\psi}=1$. Thus, by Lemma 1 , in order to prove that their Gauss sums are equal it is sufficient to verify that $T(\chi, 1)=T(\psi, 1)$. We have $\gamma_{\chi}=2$ while $\gamma_{\psi}=2+2 \cdot 3^{k-2}$, therefore $T(\chi, 1)=T(\psi, 1)$ means that

$$
\sum_{l=1}^{3^{k-1}} \zeta_{3^{k}}^{3 l \gamma_{\chi}+2^{2 l}-1}=\sum_{l=1}^{3^{k-1}} \zeta_{3^{k}}^{3 l \gamma_{\psi}+2^{2 l}-1},
$$

i.e.,

$$
\sum_{l=1}^{3^{k-1}} \zeta_{3^{k}}^{f_{0}(l)}=\sum_{l=1}^{3^{k-1}} \zeta_{3^{k}}^{f_{1}(l)}
$$

Clearly, the values of such sums are not modified by a shift $l \rightarrow l+l_{0}$, hence equality follows from (2.9) after shifting the left hand side. Note that a similar argument provides also a couple of distinct odd primitive characters with the same Gauss sum.

We finally deal with the case $q=2^{k}$ with $k \geq 6$ and prove the following

Proposition 3. Let $q=2^{k}$ with $k \geq 6$ and $\zeta_{q}$ be a primitive $q$-th root of unity. Then there exist two distinct primitive characters $\chi, \psi(\bmod q)$ with $s\left(\chi, \zeta_{q}\right)=s\left(\psi, \zeta_{q}\right)$.

The proof in this case is rather computational and requires several preliminary results. Denoting by $\beta$ the odd integer, unique $(\bmod q)$, such that $\zeta_{q}=e\left(\beta / 2^{k}\right)$, the 
Gauss sum associated with the primitive character $\chi_{\alpha, \beta}$ (see at the beginning of this section) can be written as

$$
\begin{aligned}
\tau\left(\chi_{\alpha, \beta}, \zeta_{q}\right) & =\sum_{u=1}^{2} \sum_{v=1}^{2^{k-2}} \chi_{\alpha, \beta}\left((-1)^{u} 5^{v}\right) e\left(\beta(-1)^{u} 5^{v} / 2^{k}\right) \\
& =(-1)^{\alpha} \sum_{v=1}^{2^{k-2}} e\left(\beta\left(4 v-5^{v}\right) / 2^{k}\right)+\sum_{v=1}^{2^{k-2}} e\left(\beta\left(4 v+5^{v}\right) / 2^{k}\right) \\
& =(-1)^{\alpha} \overline{\zeta_{q}} \sum_{v=1}^{2^{k-2}} \zeta_{q}^{f_{+}(v)}+\zeta_{q} \sum_{v=1}^{2^{k-2}} \zeta_{q}^{f_{-}(v)},
\end{aligned}
$$

where

$$
f_{+}(v)=4 v-5^{v}+1, \quad f_{-}(v)=4 v+5^{v}-1 .
$$

The following lemma collects a list of useful identities satisfied by the functions $f_{ \pm}(v)$.

Lemma 2. The following facts hold true:

i) $f_{+}(v)+f_{-}(v)=8 v$ for every $v$,

ii) $f_{+}(v) \equiv 0(\bmod 16)$ for every $v$,

iii) $f_{-}(v) \equiv 8(\bmod 64)$ for every odd $v$,

iv) $f_{+}\left(v+2^{k-3}\right) \equiv f_{+}(v)\left(\bmod 2^{k}\right)$ for every $v$, for $k \geq 3$,

v) $f_{+}\left(v+2^{k-3}\right) \equiv f_{+}(v)+2^{k}\left(\bmod 2^{k+1}\right)$ for every $v$, for $k \geq 4$,

vi) $f_{-}\left(v+2^{k-4}\right) \equiv f_{-}(v)\left(\bmod 2^{k}\right)$ for every $v$, for $k \geq 5$,

vii) $f_{-}\left(v+2^{k-4}\right) \equiv f_{-}(v)+2^{k}\left(\bmod 2^{k+1}\right)$ for every odd $v$, for $k \geq 6$.

Proof. By induction on $k$ we have

$$
\begin{array}{ll}
5^{2^{k-3}} \equiv 1+2^{k-1}\left(\bmod 2^{k}\right) & \text { for all } k \geq 3, \\
5^{2^{k-3}} \equiv 1+2^{k-1}+2^{k}\left(\bmod 2^{k+1}\right) & \text { for all } k \geq 4, \\
5^{2^{k-4}} \equiv 1+2^{k-2}+2^{k-1}+2^{k}\left(\bmod 2^{k+1}\right) & \text { for all } k \geq 6 .
\end{array}
$$

Now we prove each claim. i) is trivial. ii) is clearly true when $v=0$, and by induction on $v$ we have $(\bmod 16)$

$$
\begin{aligned}
0 & \equiv 5 f_{+}(v)=20 v-5^{v+1}+5 \equiv 4 v-5^{v+1}+5=4(v+1)-5^{v+1}+1 \\
& =f_{+}(v+1) .
\end{aligned}
$$


iii) Clearly $f_{-}(1) \equiv 8(\bmod 64)$. Let $v$ be odd and by induction on $v$ we assume that $f_{-}(v) \equiv 8(\bmod 64)$. Multiplying by $5^{2}$ we have $(\bmod 64)$

$$
\begin{aligned}
8 & \equiv 5^{2} \cdot 8 \equiv 5^{2} f_{-}(v)=5^{2} \cdot 4 v+5^{v+2}-5^{2} \\
& =4(v+2)+5^{v+2}-1+32(3 v-1) \equiv f_{-}(v+2) .
\end{aligned}
$$

iv) By (2.11) we have

$$
\begin{aligned}
f_{+}\left(v+2^{k-3}\right) & =4 v+2^{k-1}-5^{v+2^{k-3}}+1 \\
& \equiv 4 v+2^{k-1}-5^{v}\left(2^{k-1}+1\right)+1 \\
& =f_{+}(v)+2^{k-1}\left(1-5^{v}\right) \equiv f_{+}(v)\left(\bmod 2^{k}\right) .
\end{aligned}
$$

v) Note that

$$
f_{+}\left(v+2^{k-3}\right)=4 v+2^{k-1}-5^{v+2^{k-3}}+1=f_{+}(v)+2^{k-1}-5^{v}\left(5^{2^{k-3}}-1\right),
$$

thus recalling that $5^{y} \equiv 1(\bmod 4)$ for every $y$, by $(2.12)$ we have for some integer $h$ that

$$
\begin{aligned}
f_{+}\left(v+2^{k-3}\right) & \equiv f_{+}(v)+2^{k-1}-(1+4 h)\left(1+2^{k-1}+2^{k}-1\right) \\
& \equiv f_{+}(v)+2^{k}\left(\bmod 2^{k+1}\right) .
\end{aligned}
$$

vi) The claim follows from i) and v).

vii) Note that

$$
f_{-}\left(v+2^{k-4}\right)=4 v+2^{k-2}+5^{v+2^{k-4}}-1=f_{-}(v)+2^{k-2}+5^{v}\left(5^{2^{k-4}}-1\right),
$$

hence recalling that $5^{y} \equiv 5(\bmod 8)$ for every odd $y$, by (2.13) we have for some integer $h$ that

$$
\begin{aligned}
f_{-}\left(v+2^{k-4}\right) & \equiv f_{-}(v)+2^{k-2}+(1+4+8 h)\left(1+2^{k-2}+2^{k-1}+2^{k}-1\right) \\
& \equiv f_{-}(v)+2^{k-2}+2^{k-2}+2^{k-1}+2^{k}+2^{k} \\
& \equiv f_{-}(v)+2^{k}\left(\bmod 2^{k+1}\right),
\end{aligned}
$$

and the lemma follows.

Lemma 3. Let $k \geq 4$. The function $f_{+}(v)\left(\bmod 2^{k}\right)$ assumes each value exactly twice when $v=1, \ldots, 2^{k-3}$. Moreover, for such $v$ the range of $f_{+}(v)\left(\bmod 2^{k}\right)$ coincides with the numbers $16 \ell, \ell=1, \ldots, 2^{k-4}$, each one with multiplicity 2 .

Proof. We first prove that every value of $f_{+}(v)$ is assumed at least twice. A trivial computation shows that

$$
0=f_{+}(1) \equiv f_{+}(2)\left(\bmod 2^{4}\right)
$$


proving our claim for $k=4$. Now we proceed by induction. Let $k \geq 4$ and let $x_{0}, y_{0} \in\left\{1, \ldots, 2^{k-3}\right\}, x_{0} \neq y_{0}$, satisfy $f_{+}\left(x_{0}\right) \equiv f_{+}\left(y_{0}\right)\left(\bmod \overline{2}^{k}\right)$. We distinguish two cases.

Case 1. Suppose that $f_{+}\left(x_{0}\right) \not \equiv f_{+}\left(y_{0}\right)\left(\bmod 2^{k+1}\right)$. Then $f_{+}\left(x_{0}\right) \equiv f_{+}\left(y_{0}\right)+$ $2^{k}\left(\bmod 2^{k+1}\right)$ and hence $\left.\mathrm{v}\right)$ of Lemma 2 shows that $\left(x_{0}+2^{k-3}, y_{0}\right)$ and $\left(x_{0}, y_{0}+\right.$ $\left.2^{k-3}\right)$ are solutions of $f_{+}(x) \equiv f_{+}(y)\left(\bmod 2^{k+1}\right)$.

Case 2. Suppose that $f_{+}\left(x_{0}\right) \equiv f_{+}\left(y_{0}\right)\left(\bmod 2^{k+1}\right)$. Then by v) of Lemma 2 we obtain $f_{+}\left(x_{0}+2^{k-3}\right) \equiv f_{+}\left(x_{0}\right)+2^{k} \equiv f_{+}\left(y_{0}\right)+2^{k} \equiv f_{+}\left(y_{0}+2^{k-3}\right)(\bmod$ $\left.2^{k+1}\right)$, hence $\left(x_{0}+2^{k-3}, y_{0}+2^{k-3}\right)$ is also a solution $\left(\bmod 2^{k+1}\right)$.

Let now $x \in\left\{1, \ldots, 2^{k-2}\right\}$. If $x \leq 2^{k-3}$ then write $x_{0}=x$ and there exists $y_{0} \in\left\{1, \ldots, 2^{k-3}\right\}, y_{0} \neq x_{0}$, such that $f_{+}\left(x_{0}\right) \equiv f_{+}\left(y_{0}\right)\left(\bmod 2^{k}\right)$. If we are in Case 1 then $f_{+}\left(x_{0}\right) \equiv f_{+}\left(y_{0}+2^{k-3}\right)\left(\bmod 2^{k+1}\right)$ and hence the value $f_{+}(x)(\bmod$ $\left.2^{k+1}\right)$ is attained at least twice, while if we are in Case 2 then $f_{+}\left(x_{0}\right) \equiv f_{+}\left(y_{0}\right)$ $\left(\bmod 2^{k+1}\right)$ and again the value $f_{+}(x)\left(\bmod 2^{k+1}\right)$ is attained at least twice. If $2^{k-3}<x \leq 2^{k-2}$ we write $x_{0}=x-2^{k-3}$ and again there exists $y_{0} \in\left\{1, \ldots, 2^{k-3}\right\}$, $y_{0} \neq x_{0}$, such that $f_{+}\left(x_{0}\right) \equiv f_{+}\left(y_{0}\right)\left(\bmod 2^{k}\right)$. Then we repeat the same argument, using the solution $\left(x_{0}+2^{k-3}, y_{0}\right)$ in Case 1 and the solution $\left(x_{0}+2^{k-3}, y_{0}+2^{k-3}\right)$ in Case 2, thus proving that the value $f_{+}(x)$ is assumed at least twice in this case as well.

Now we prove that each value is assumed exactly twice. When $k=4$ the claim is true. Assume that there exists a minimal value $\bar{k}>4$ for which there are distinct integers $u_{0}, v_{0}, w_{0} \in 1, \ldots, 2^{\bar{k}-3}$ with $f_{+}\left(u_{0}\right) \equiv f_{+}\left(v_{0}\right) \equiv f_{+}\left(w_{0}\right)\left(\bmod 2^{\bar{k}}\right)$. Write

$$
\tilde{u}_{0}= \begin{cases}u_{0} & \text { if } u_{0} \leq 2^{\bar{k}-4}, \\ u_{0}-2^{\bar{k}-4} & \text { if } u_{0}>2^{\bar{k}-4},\end{cases}
$$

and similarly for $\tilde{v}_{0}$ and $\tilde{w}_{0}$. By iv) of Lemma 2 we have that $f_{+}\left(\tilde{u}_{0}\right) \equiv f_{+}\left(u_{0}\right)$ $\left(\bmod 2^{\bar{k}-1}\right)$, hence $f_{+}\left(u_{0}\right) \equiv f_{+}\left(v_{0}\right)\left(\bmod 2^{\bar{k}}\right)$ implies that $f_{+}\left(\tilde{u}_{0}\right) \equiv f_{+}\left(\tilde{v}_{0}\right)(\bmod$ $\left.2^{\bar{k}-1}\right)$. Hence $f_{+}\left(\tilde{u}_{0}\right) \equiv f_{+}\left(\tilde{v}_{0}\right) \equiv f_{+}\left(\tilde{w}_{0}\right)\left(\bmod 2^{\bar{k}-1}\right)$. From the minimality of $\bar{k}$ we obtain that $\tilde{u}_{0}, \tilde{v}_{0}, \tilde{w}_{0}$ cannot be all distinct; let $\tilde{u}_{0}=\tilde{v}_{0}$, say. The definition of such numbers implies that either $u_{0}=v_{0}$, or $u_{0}+2^{\bar{k}-4}=v_{0}$, or $u_{0}=v_{0}+2^{\bar{k}-4}$. The first case is ruled out by our assumption, and the second and third cases are treated similarly (it is sufficient to change $u_{0}$ with $v_{0}$ ), so we may assume that $u_{0}=v_{0}+2^{\bar{k}-4}$. By v) of Lemma 2 we get

$$
f_{+}\left(u_{0}\right) \equiv f_{+}\left(v_{0}+2^{\bar{k}-4}\right) \equiv f_{+}\left(v_{0}\right)+2^{\bar{k}-1}\left(\bmod 2^{\bar{k}}\right)
$$

which contradicts our assumption that $f_{+}\left(u_{0}\right) \equiv f_{+}\left(v_{0}\right)\left(\bmod 2^{\bar{k}}\right)$, thus proving the first claim.

By ii) of Lemma 2 we know that the values of $f_{+}(v)$ are of the form $16 \ell$, hence the number of distinct values of $f_{+}(v)\left(\bmod 2^{k}\right)$ is at most $2^{k} / 16=2^{k-4}$. On the 
other hand, by the periodicity $\left(\bmod 2^{k}\right)$ expressed by iv) of Lemma 2 and the fact that each value of $f_{+}(v)$ with $1 \leq v \leq 2^{k-3}$ is attained exactly twice, we have that the distinct values of $f_{+}(v)\left(\bmod 2^{k}\right)$ with $1 \leq v \leq 2^{k-2}$ are at least $2^{k-2} / 4=2^{k-4}$. Therefore the values of $f_{+}(v)\left(\bmod 2^{k}\right)$ are the numbers $16 \ell$ with $1 \leq \ell \leq 2^{k-4}$, and the lemma follows.

The argument proving Lemma 3 shows also that when $1 \leq v_{1}, v_{2} \leq 2^{k-3}$ and $f_{+}\left(v_{1}\right) \equiv f_{+}\left(v_{2}\right)\left(\bmod 2^{k}\right)$ then $v_{1}$ and $v_{2}$ cannot have the same parity. As a consequence we have the following

Lemma 4. Let $k \geq 4$. The function $f_{+}(v)\left(\bmod 2^{k}\right)$ assumes each value exactly once when $v \in\left\{1, \ldots, 2^{k-3}\right\}$ runs over odd integers. Moreover, for such $v$ the range of $f_{+}(v)\left(\bmod 2^{k}\right)$ coincides with the numbers $16 \ell, \ell=1, \ldots, 2^{k-4}$, each one with multiplicity 1 .

The following lemma gives a similar result for the function $f_{-}(v)$.

Lemma 5. Let $k \geq 6$. The function $f_{-}(v)\left(\bmod 2^{k}\right)$ assumes each value exactly twice when $v \in\left\{1, \ldots, 2^{n-4}\right\}$ runs over odd integers. Moreover, for such $v$ the range of $f_{-}(v)\left(\bmod 2^{k}\right)$ coincides with the numbers $8+64 \ell, \ell=1, \ldots, 2^{k-6}$, each one with multiplicity 2.

Proof. The proof is similar to the proof of Lemma 3, using iii), vi) and vii) of Lemma 2 instead of ii), iv) and v) of the same lemma. We leave the details to the interested reader.

Proof of Proposition 3. A direct computation shows that the two distinct primitive characters $\chi=\chi_{\alpha, 4+\beta}$ and $\psi=\chi_{\alpha, 4+8+\beta}$ (which are even when $\alpha=0$ and odd when $\alpha=1$ ) have $\tau\left(\chi, e\left(\beta / 2^{6}\right)\right)=\tau\left(\psi, e\left(\beta / 2^{6}\right)\right)$ for every odd $\beta$ (see Table 4), hence our claim for $n=6$ is proved.

If $q=2^{k}$ with $k \geq 7$ write $\chi=\chi_{\alpha, \beta}$ and $\psi=\chi_{\alpha, \beta+\beta 2^{k-3}}$ where, again, $\alpha=0,1$ and $\beta$ is the odd integer such that $\zeta_{q}=e(\beta / q)$. We prove that $\tau\left(\chi, \zeta_{q}\right)=\tau\left(\psi, \zeta_{q}\right)$. In fact

$$
\begin{aligned}
\tau\left(\psi, \zeta_{q}\right)=(-1)^{\alpha} \sum_{v=1}^{2^{k-2}} e\left(\left(4 \beta\left(1+2^{k-3}\right) v-\beta 5^{v}\right) / q\right) \\
+\sum_{v=1}^{2^{k-2}} e\left(\left(4 \beta\left(1+2^{k-3}\right) v+\beta 5^{v}\right) / q\right) \\
=(-1)^{\alpha} \bar{\zeta}_{q} \sum_{v=1}^{2^{k-2}}(-1)^{v} \zeta_{q}^{f_{+}(v)}+\zeta_{q} \sum_{v=1}^{2^{k-2}}(-1)^{v} \zeta_{q}^{f_{-}(v)}
\end{aligned}
$$


and hence, thanks to (2.10),

$$
\tau\left(\chi, \zeta_{q}\right)-\tau\left(\psi, \zeta_{q}\right)=(-1)^{\alpha} 2 \bar{\zeta}_{q} \sum_{\substack{v=1 \\ v \text { odd }}}^{2^{k-2}} \zeta_{q}^{f_{+}(v)}+2 \zeta_{q} \sum_{\substack{v=1 \\ v \text { odd }}}^{2^{k-2}} \zeta_{q-}^{f_{-}(v)}
$$

Therefore, the $2^{k-3}$-periodicity of $f_{+}(v)\left(\bmod 2^{k}\right)$ in iv) of Lemma 2 and Lemma 4 give

$$
\sum_{\substack{v=1 \\ v \text { odd }}}^{2^{k-2}} \zeta_{q}^{f_{+}(v)}=2 \sum_{\substack{v=1 \\ v \text { odd }}}^{2^{k-3}} \zeta_{q}^{f_{+}(v)}=2 \sum_{\ell=1}^{2^{k-4}} e\left(\beta 16 \ell / 2^{k}\right)=2 \sum_{\ell=1}^{2^{k-4}} e\left(\beta \ell / 2^{k-4}\right)=0
$$

when $k \geq 5$. Analogously, the $2^{k-4}$-periodicity of $f_{-}(v)\left(\bmod 2^{k}\right)$ in vi) of Lemma 2 and Lemma 5 give

$$
\begin{aligned}
\sum_{\substack{v=1 \\
v \text { odd }}}^{2^{k-2}} \zeta_{q}^{f_{-}(v)} & =4 \sum_{\substack{v=1 \\
v \text { odd }}}^{2^{k-4}} \zeta_{q}^{f_{-}(v)}=8 \sum_{\ell=1}^{2^{k-6}} e\left(\beta(8+64 \ell) / 2^{k}\right) \\
& =8 \zeta_{q}^{8} \sum_{\ell=1}^{2^{k-6}} e\left(\beta \ell / 2^{k-6}\right)=0
\end{aligned}
$$

when $k \geq 7$. Proposition 3 follows since $\chi$ and $\psi$ are distinct primitive characters with the same parity.

The proof of Theorem 2 is now a simple consequence of Propositions 1, 2 and 3. In fact, by the special case $\zeta_{q}=e(1 / q)$ of Proposition 1 we have that if $q \in \mathbb{Q}$ then the map $s_{q}$ is injective. Viceversa, assume that $q \not \equiv 2(\bmod 4)$ does not belong to $\mathbb{Q}$. Then, writing $q=2^{a} 3^{b} m$ with $(m, 6)=1$, we have the following cases:

(1) $m$ is not square-free and $a, b$ are arbitrary with $a \neq 1$;

(2) $m$ is square-free and both conditions (a) and (b) are not satisfied.

In case (1) we can write $q=q_{1} q_{2}$ with $\left(q_{1}, q_{2}\right)=1$ and $q_{1}=p^{k}$ for some prime $p \geq 5$ and $k \geq 2$. Thanks to (2.1), given primitive characters $\chi_{1}\left(\bmod q_{1}\right)$ and $\chi_{2}$ $\left(\bmod q_{2}\right)$ let $\zeta_{q_{1}}$ and $\zeta_{q_{2}}$ be the primitive roots (not depending on $\chi_{1}$ and $\chi_{2}$ ) such that

$$
\tau\left(\chi_{1} \chi_{2}, e(1 / q)\right)=\tau\left(\chi_{1}, \zeta_{q_{1}}\right) \tau\left(\chi_{q_{2}}, \zeta_{q_{2}}\right) .
$$

Now fix the primitive character $\chi_{2}\left(\bmod q_{2}\right)$. By Proposition 2, there exist two distinct primitive characters $\chi_{1}$ and $\chi_{1}^{\prime}\left(\bmod q_{1}\right)$ with the same signature with respect to $\zeta_{q_{1}}$. Finally, consider the distinct primitive characters $\chi=\chi_{1} \chi_{2}$ and $\psi=\chi_{1}^{\prime} \chi_{2}(\bmod q)$. Clearly $\chi$ and $\psi$ have the same parity, and thanks to (2.14) we have

$$
\tau(\chi)=\tau\left(\chi_{1}, \zeta_{q_{1}}\right) \tau\left(\chi_{q_{2}}, \zeta_{q_{2}}\right)=\tau\left(\chi_{1}^{\prime}, \zeta_{q_{1}}\right) \tau\left(\chi_{q_{2}}, \zeta_{q_{2}}\right)=\tau(\psi) .
$$


Thus the map $s_{q}$ is not injective in this case.

The argument in case (2) is similar. In this case we have either $a \geq 6$, or $b \geq 3$, or $a \in\{4,5\}$ and $b=2$. The first two subcases are treated in a similar way, using Proposition 3 and Proposition 2, respectively. Indeed, in both cases we can write $q=q_{1} q_{2}$ with $\left(q_{1}, q_{2}\right)=1$ and $q_{1}=2^{a}$ or $q_{1}=3^{b}$, thus there exist two distinct primitive characters $\chi_{1}$ and $\chi_{1}^{\prime}\left(\bmod q_{1}\right)$ with the same signature with respect to the primitive root $\zeta_{q_{1}}$, and the non-injectivity of the map $s_{q}$ follows again. Finally, if $a \in\{4,5\}$ and $b=2$, we note that modulo $2^{4}, 2^{5}$ and $3^{2}$ and for every primitive root, there exist two distinct primitive characters with the same Gauss sum but with different parity, see Tables 2, 3 and 5. Hence, multiplying such characters in a suitable way we get two distinct primitive characters $\left(\bmod q_{1}\right), q_{1}=2^{a} 3^{2}$, with the same signature, and we proceed as before thus showing that the map $s_{q}$ is not injective in this subcase as well.

Clearly, the above arguments give exactly the same characterization of Theorem 2 for the integers $q$ such that the map $s_{q, \zeta_{q}}$ is injective. Indeed we have

Theorem 4. Let $\zeta_{q}$ be a primitive $q$-th root of unity. Then the map $s_{q, \zeta_{q}}$ is injective if and only if $q \in \mathbb{Q}$.

We also remark that the same arguments can be used to give the following characterization of the integers $q$ for which the map $s_{q, \zeta_{q}}^{\prime}$ sending primitive $\chi$ 's to $\tau\left(\chi, \zeta_{q}\right)$ is injective.

Theorem 5. Let $\zeta_{q}$ be a primitive $q$-th root of unity. Then the map $s_{q, \zeta_{q}}^{\prime}$ is injective if and only if $q=2^{a} m$ with $m$ odd square-free and $a \in\{0,2,3\}$.

\section{References}

[1] A. Baker, B. J. Birch, and E. A. Wirsing, On a problem of Chowla. J. Number Theory 5 (1973), 224-236. Zbl 0267.10065 MR 0340203466

[2] B. C. Berndt, R. J. Evans, and K. S. Williams, Gauss and Jacobi sums. Canad. Math. Soc. Ser. Monogr. Adv. Texts, John Wiley \& Sons, New York 1998. Zbl 0906.11001 MR 1625181468

[3] J. W. Cogdell and I. I. Piatetski-Shapiro, Converse theorems for $\mathrm{GL}_{n}$ and their application to liftings. In Cohomology of arithmetic groups, L-functions and automorphic forms, ed. by T. N. Venkataramana, Tata Inst. Fund. Res. Studies in Math. 15, Narosa Publishing House, New Delhi, 2001, 1-34. Zbl 1039.11030 MR 1986092464

[4] J. B. Conrey and D. W. Farmer, An extension of Hecke's converse theorem. Int. Math. Res. Notices (1995), 445-463. Zbl 0849.11042 MR 1360623464 
[5] L. Ehrenpreis and T. Kawai, Poisson's summation formula and Hamburger's theorem. Publ. Res. Inst. Math. Sci. 18 (1982), 413-426. Zbl 0499.10042 MR 0677271466

[6] M. I. Gurevič, Determining L-series from their functional equations. Math. USSR Sb. 14 (1971), 537-553. Zbl 0225.12007 MR 0280497466

[7] H. Hamburger, Über die Riemannsche Funktionalgleichung der $\zeta$-Funktion. Math. Z. 10 (1921), 240-254; 11 (1922), 224-245; 13 (1922), 283-311. JFM 48.1210.03 MR 1544495463

[8] E. Hecke, Lectures on Dirichlet series, modular functions and quadratic forms. Vanderhoeck \& Ruprecht, Göttingen 1983. Zbl 0507.10015 MR 0693092463

[9] J. Kaczorowski and A. Perelli, On the structure of the Selberg class, I: $0 \leq d \leq 1$. Acta Math. 182 (1999), 207-241. Zbl 1126.11335 MR 1710182 464, 465, 466

[10] J. Kaczorowski and A. Perelli, On the structure of the Selberg class, IV: basic invariants. Acta Arith. 104 (2002), 97-116. Zbl 0996.11053 MR 1914246464

[11] J.-L. Mauclaire, Sommes de Gauss modulo $p^{\alpha}$. I; II. Proc. Japan Acad. Ser. A 59 (1983), 109-112; 59 (1983), 161-163. Zbl 0516.10028 MR 0711310 MR 0711325468

[12] G. Molteni, Multiplicity results for the functional equation of the Dirichlet $L$-functions. Preprint 2009. 467

[13] G. Molteni, Multiplicity results for the functional equation of the Dirichlet $L$-functions: case $p=2$. Preprint 2009. 467

[14] R. W. K. Odoni, On Gauss sums $\left(\bmod p^{n}\right), n \geq 2$. Bull. London Math. Soc. 5 (1973), 325-327. Zbl 0269.10020 MR 0327678468

[15] I. Piatetski-Shapiro and R. Raghunathan, On Hamburger's theorem. Amer. Math. Soc. Transl. (2) 169 (1995), 109-120. Zbl 0843.11041 MR 1364456 463, 464

[16] E. C. Titchmarsh, The theory of the Riemann Zeta-function. Second edition, Oxford University Press, New York 1986. Zbl 0601.10026 MR 0882550463

[17] A. Weil, Über die Bestimmung Dirichletscher Reihen durch Funktionengleichungen. Math. Ann. 168 (1967), 149-156. Zbl 0158.08601 MR 0207658464

[18] A. Yoshimoto, On a generalization of Hamburger's theorem. Nagoya Math. J. 98 (1985), 67-76. Zbl 0547.12011 MR 0792771466

Received April 30, 2008

Jerzy Kaczorowski, Faculty of Mathematics and Computer Science, Adam Mickiewicz University, ul. Umultowska 87, 61-614 Poznań, Poland

E-mail: kjerzy@amu.edu.pl

Giuseppe Molteni, Dipartimento di Matematica, Università di Milano, via Saldini 50, 20133 Milano, Italy

E-mail: giuseppe.molteni1@unimi.it

Alberto Perelli, Dipartimento di Matematica, Università di Genova, via Dodecaneso 35, 16146 Genova, Italy

E-mail: perelli@dima.unige.it 